CLASSIC PAPER

\title{
Effectiveness of a simulator in training anesthesiology residents*
}

\author{
S Abrahamson, J S Denson, R M Wolf
}

Qual Saf Health Care 2004;13:395-399

The educational potential of a computer-controlled patient simulator was tested by the University of Southern California School of Medicine. The results of the experiment suggest unequivocally that there is a twofold advantage to the use of such a simulator in training anesthesiology residents in the skill of endotracheal intubation: (a) residents achieve proficiency levels in a smaller number of elapsed days of training, thus effecting a saving of time in the training of personnel, and (b) residents achieve a proficiency level in a smaller number of trials in the operating room, thus posing significantly less threat to patient safery. The small number of subjects in the study and the large within-group variability were responsible for a lack of statistical significance in 4 of 6 of the analyses performed; however, all differences were substantial and in the hypothesized direction. Thus, despite the narrowly circumscribed tasks to be learned by the experimental subjects, the findings suggest that the use of simulation devices should be considered in planning for future education and training not only in medicine but in other health care professions as well.

See end of article for authors' affiliations
$\mathrm{T}$ he use of simulation in medical education is increasing in frequency and in sophistication. Perhaps the most advanced simulation tool yet constructed is a computer-controlled anthropometric manikin developed by the University of Southern California, a device now known popularly as "Sim One". This paper reports the results of an experiment testing the effectiveness of Sim One in the training of anesthesiology residents in the maneuver of endotracheal intubation.

Sim One is lifelike in appearance, having a plastic skin which resembles that of a real human being in color and texture; its configuration is that of a patient lying on an operating table, left arm extended and ready for intravenous injection, right arm fitted with blood pressure cuff, and chest wall having a stethoscope taped over the approximate location of the heart. It breathes; has a heart beat, temporal and carotid pulse (all synchronized), and blood pressure; opens and closes its mouth; blinks its eyes; and responds to four intravenously administered drugs and two gases (oxygen and nitrous oxide) administered through mask or tube. The physiologic responses to what is done to him are in real time and occur "automatically" as part of a computer program.

The entire maneuver of endotracheal intubation can be performed in exactly the manner in which it is done in the operating room. At the outset, oxygen is administered through maskand-bag, following which there is an injection of sodium pentothal. Approximately 20 seconds later, Sim One's eyes close and unconsciousness is simulated. Succinyl choline is injected, bringing about complete relaxation of muscles and cessation of breathing. Through the use of a laryngoscope, a tube is inserted into the trachea and oxygen and nitrous oxide are then administered by pressure on the reservoir bag.

The assumption of several educational "advantages" led to the statement of hypothesis underlying this study. The first potential advantage is that the use of the simulator allows for a planned and gradual increase in the difficulty of the problems to be solved by the anesthesiology resident instead of his performing new tasks in the operating room as the necessity for them arises. Second, the use of the simulator permits almost unlimited repetition of any phase of the procedures to be learned. Third, the resident can obtain immediate feedback on his own performance in the form of a printout of all acts performed in the order in which they took place and with the exact time elapsed also noted. Finally, each learner proceeds at his own rate.

It was hypothesized that residents trained on the simulator would achieve predesignated criterion levels of performance in less time and with fewer operating room trials than residents not permitted to use the simulator.

\section{STUDY DESIGN}

The anesthesiology resident training program at the Los Angeles County General Hospital is one of the largest in the country: at any given time there are more than 24 residents in training. It was anticipated that 12 new residents would arrive after 1 July 1967. These individuals were to be paired for purpose of study, one of each pair to be afforded training on the simulator and the other to engage in the usual training procedures without the benefit of experience on the simulator. The decision as to which member of each pair would be given training on the simulator was made by a flip of a coin. The pairing was accomplished over a period of time as each resident arrived (between the late spring of 1967

${ }^{*}$ This is a reprint of a paper that appeared in Journal of Medical Education, 1969, Volume 44, pages 515-9. 


\begin{tabular}{|c|c|c|c|}
\hline Mastery criterion & $\begin{array}{l}\text { Experimental } \\
(\mathrm{N}=5)\end{array}$ & $\begin{array}{l}\text { Control } \\
(\mathrm{N}=5)\end{array}$ & $\begin{array}{l}F \text { ratio } \\
(\mathrm{N}=5)\end{array}$ \\
\hline Trials to 4 consecutive & 9.6 & 18.6 & 2.16 \\
\hline Days to 4 consecutive & 17.0 & 22.8 & 0.46 \\
\hline Trials to 7 of 8 & 28.4 & 43.8 & 3.25 \\
\hline Days to 7 of 8 & 42.8 & 57.8 & 1.41 \\
\hline Trials to 9 of 10 & 30.0 & 59.8 & $38.30^{*}$ \\
\hline Days to 9 of 10 & 45.6 & 77.0 & $9.20^{* *}$ \\
\hline
\end{tabular}

and the fall of 1967). Eventually, only five pairs were used because two of the residents had had extensive experience in endotracheal intubation during the year prior to beginning their residency training, each having accomplished more than 150 intubations. The five residents assigned to Sim One completed their training on the simulator in varying periods of time, ranging from 5.5 hours to 9.5 hours over a 2 week period. Had the visits to Sim One not required a considerable amount of cross-city travel, each resident could have completed the exercise in 2 or 3 days.

The design of the study necessitated some measure of proficiency in endotracheal intubation. The plan was to observe each of the 10 residents in the operating room every time he was required to participate in endotracheal intubation. In addition, similar observation was to be made of the five simulator-trained residents during their training runs on Sim One. Comparisons between simulator-trained residents and those in the control group were to be made on the basis of elapsed time from date of arrival in the program to date of performance at a professional level of proficiency.

The investigators used as the source of data the official anesthesia charts of the hospital. Each anesthesiologist is obliged to maintain a complete record of the administration of anesthesia during surgery procedures as part of the official hospital records. These anesthesia charts include a sufficient amount of information concerning his proficiency.

The anesthesia charts of all endotracheal intubations performed by the 10 residents in the study were subjected to critical appraisal. The names of the resident, the patient, and other personnel, as well as the date of the intubation were completely concealed and each chart was submitted to the Department of Anesthesiology with this question posed: "On the basis of what you see on this chart, would you be willing to trust the anesthesiology resident in an operating room without supervision?" The raters gave the charts a "plus" rating for professionally acceptable performance and a "minus" rating for unacceptable performance. In all, 1220 charts were reviewed and rated.
Following completion of the rating process, the anesthesia charts were sorted according to residents' names and arranged in chronological order from the date of the first intubation completed by the resident to that of the last intubation within the time period of the study. Three criterion levels of performance were selected: the number of operating room trials necessary for a resident to achieve 4, 7 of 8 , and 9 of 10 consecutive plus ratings. As a second variable the investigators examined the number of elapsed days between the first endotracheal intubation in the operating room and achievement of each of these three criterion levels.

\section{FINDINGS}

The data were subjected to analysis of variance ( $\mathrm{F}$ ratio) using a randomized block design for all analyses. Despite the small number of subjects, statistical significance was tested. The results are presented in table 1 which lists the mean number of trials and days necessary for the residents to achieve each of the criterion levels of performance and are summarized in fig 1 .

The smallest differences to be noted are in the number of elapsed days and number of trials required to achieve four consecutive plus ratings. As the more rigorous criteria are applied ( 7 of 8 and 9 of 10), the differences tend to be larger and a 0.01 level of statistical significance is reached for the last performance criterion ( 9 of 10 consecutive plus ratings). Furthermore, the difference between the mean number of elapsed days required to meet this criterion is significant at the 0.05 level.

\section{DISCUSSION}

Despite the fact that four of the six analyses failed to reach a level of statistical significance, the investigators conclude that there is a time advantage in using this computer-controlled patient simulator in the training of anesthesiology residents. The results of the study are all in the hypothesized direction-that is, residents using the simulator reached
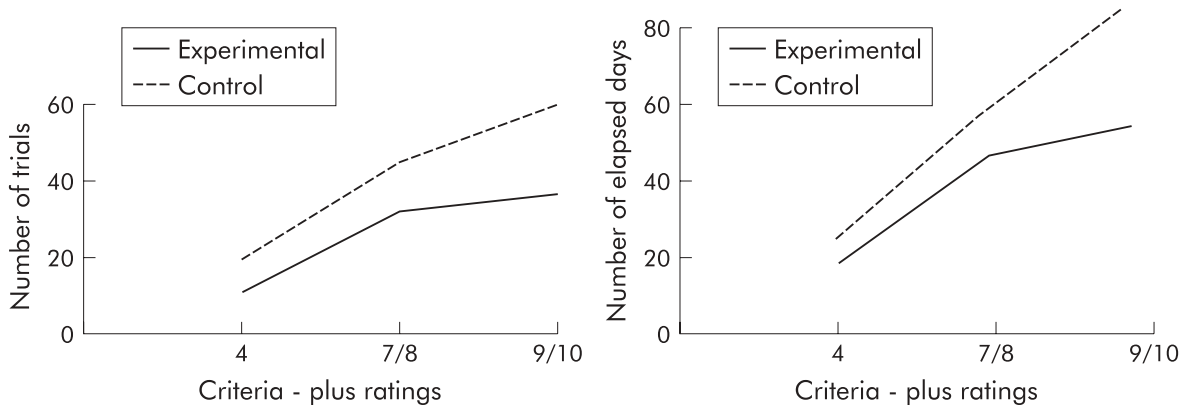

Figure 1 The graph on the left shows the mean number of trials and the one on the right the mean number of elapsed days required by five experimental and five control subjects to reach three prescribed criterion levels of performance. 
accepted professional levels of performance in fewer elapsed days and in a smaller number of trials in the operating room than did residents who did not have a training period on the simulator.

The failure to reach statistical significance can be accounted for by the large within-group variability and the small number of subjects involved in the study. The differences in means for each of the criterion levels are substantial, if not significant. But the figures, perhaps, do not tell the whole story. For instance, a minus rating signifies that some aspect or aspects of total performance were so lacking in quality that an experienced anesthesiologist stated that he would not trust this man in the operating room without supervision. Thus, potential discomfort or even harm is posed to the patient during that endotracheal intubation. Significantly less threat to patient welfare is posed by residents who have been trained on the patient simulator. The mean number of trials necessary for simulator-trained anesthesiology residents to achieve four consecutive ratings is 9.6 as compared to 18.6 for those not trained on the simulator so, on average, nine more patients were posed with minor, or less minor, threat by each beginning anesthesiology resident not trained on the simulator before that resident achieved skill enough to perform four consecutive professionally acceptable endotracheal intubations. These differences grow as the criterion level becomes more exacting.

In addition, Sim One promises a significant time saving in training. Again, despite the lack of statistical significance in two analyses, the differences between experimental and control groups in the time required to reach each criterion level are all in the hypothesized direction; and the one significant result demonstrates a 22 day time saving, on average, in the achievement of 9 of 10 consecutive plus ratings - the most exacting criterion applied. Thus, by extrapolation, beginning anesthesiology residents might be expected to achieve this level of professional competence in a saving of 22 days over a period of 77 days. If all of the skills to be learned by anesthesiology residents could be taught through simulators, one can speculate that the achievement of these skills might be accomplished in less than three quarters of the time now required.

\section{Authors' affiliations}

S Abrahamson, Director, Division of Research in Medical Education, University of Southern California School of Medicine, Los Angeles, USA J S Denson, Professor of Surgery (Anesthesiology Chairman), University of Southern California School of Medicine, Los Angeles, USA R M Wolf, Department of Psychology, Teachers College-Columbia University, New York City, USA

Supported by the US Office of Education, Department of Health, Education, and Welfare, Cooperative Research Project No. D-240, Contract OE 6-10-135.

Delivered at the Conference on Research in Medical Education, 79th Annual Meeting of the Association of American Medical Colleges, Houston, Texas, 1 November 1968.

\section{COMMENTARY}

\section{WILL SIMULATION FLY IN MEDICINE AS IT HAS IN AVIATION?}

What is fascinating about the study by Abrahamson et al ${ }^{1}$ are the similarities between medicine and aviation in the technology and use of simulation 35 years ago. What is also surprising is the advancement made by aviation to integrate simulation into training over the past 35 years compared with medicine in which simulation remains relatively unchanged. Medicine is asking the question-does simulation really work and is there a place for it in medical training? It should be asking why simulation in medicine is still a stand alone device used to practice very limited tasks, with narrow integration of the devices into the training curriculum of medical professionals.

Although there are many reasons why it has not done so, medicine must become proactive and move beyond traditional methods of medical training. It needs to embrace simulation, create an effective model of training with simulation, and integrate simulation into a system design approach to training before the effectiveness of simulation can be assessed.

\section{Use of simulation in aviation}

I will briefly address the history of simulation in aviation and how it has developed and will then apply some of these aviation concepts to the study by Abrahamson et al in the context of an integrated system approach. Although I am applying what has been learned in aviation to this study, it can be applied to simulation in any of the disciplines of medicine that are developing simulations for their future training. My hope is to paint a picture of the future of medical training in which simulation will be an integral part of the training design. Simulation will not replace patient care but will enhance the quality of training.

In aviation, simulators were very limited devices 35 years ago, used to train very specific tasks to individual pilots. The aircraft was used as the primary trainer for pilots which required them to fly countless hours without income from passengers or cargo for the airline. One of the most critical tasks in aviation is the ability to land a large 200 passenger transport aircraft safely. As simulation technology advanced in the early 1970s, the psychomotor stimulus presentation by the simulators to the pilots was of sufficient fidelity to allow complete training of the landing maneuver in the simulator. At the time there was a great outcry from pilots that simulation could never take the place of aircraft for training. However, after careful testing and analysis, the simulator was proved to be as effective-if not more so-than the aircraft for training this critical maneuver as well as other technical and human factor skills. ${ }^{2-4}$ Today, the first time a pilot lands the aircraft is with 200 passengers on board with a safety check pilot in the other seat. No one could imagine using an aircraft to train today.

After this monumental event, the next big advancement was crew training. In the early 1980s most airline training focused almost exclusively on the technical aspects of flying and on an individual pilot's performance. In fact, although aircraft were certified to be flown with two or three pilots, most training in the simulator was performed with just one pilot in the seat practising very specific technical skills. The safety reporting systems and National Transportation Safety Board (NTSB) reports began to show that over $70 \%$ of the aircraft accidents and incidents were not caused by a pilot's technical skill but by lack of human factor skills. Investigations into the causes of air carrier accidents have shown that human error is a contributing factor in $60-80 \% .^{5}{ }^{6}$ Long term NASA research has shown that these events share common characteristics. Many problems encountered by flight crews have very little to do with the technical aspects of operating in a multi-person cockpit. Instead, problems are associated with poor group decision making, ineffective communication, inadequate leadership, and poor task or resource management. Pilot training programs historically did not effectively address crew management issues that are also fundamental to safe flight. The Advanced Qualification Program (AQP) was developed to allow the integration of technical skills with human factor skills to evaluate the crew 


\section{Box 1 Example of a skill group}

\section{Strategic planning}

- Proactive preparation

- Active monitoring

- Establishing specific parameters and guidelines

- Communicate and confirm agreement and understanding of plan

- Technical knowledge and experience

- Includes operational limitations and considerations in planning

- Establish limits

- Define personal and operational limits

- Alternate plan is initiated when limits are exceeded

- Communicate and confirm agreement and understanding of limits

- Contingency planning

- Establish alternatives

- Anticipate outcomes

- Communicate and confirm agreement and understanding of plan

in an operational environment created in the simulator. AQP has identified two subsections of crew resource management (CRM) or team training:

- CRM Topics and Skills which concentrates on the attitudes and behavior of crew members and the impact on flight safety.

- CRM Topics and Applied Practical Flight Management Skills and intervention tools.

These different topics and related skills require different instructional strategies. In addition, different groups or clusters of skills apply to specific issues such as error management, proceduralized forms of CRM, and autoflight management. An example of a skill group is shown in box 1 .

Although there has been further advancement in simulation, most of this development has been to integrate the technical and human performance, develop the training for the instructors and evaluators, and establish the metrics to monitor program performance. As aviation simulation moves into the 21 st century, the need for information and error management and assessment have been added to the simulation and crew performance.

\section{Application of simulation to medicine}

Some of the integration processes developed in aviation can be applied to the paper by Abrahamson et al. ${ }^{1}$ To measure the true effectiveness of simulation in training it must be integrated throughout the training period. The analysis and design of this study focused on a partial task in the subset of skills required by an anesthesiologist. However, the simulator used has greater capabilities, as described by the authors. To demonstrate the true effectiveness of this device in training, the training and measurements should move beyond this narrow technical focus and include the dynamic operational performance in the operating room.
Training and assessment of medical students in an environment in which simulation is integrated into a system approach to training would begin with part task trainers. The skills which they could teach would include intubation, administration of anesthesia and medications, monitoring skills for anesthesiologists, interfaces with the various types of anesthesia machines, and skills to manage equipment failure when it occurs.

Once these skills have been mastered, simulation moves to the next level which is to apply these skills in the operating room environment. The operating room should be based on medical team concepts. As discussed earlier, aviation has assigned $70 \%$ of the probable cause of accidents and incidents to the role played by interpersonal, cultural, and other nontechnical elements. These factors have a significant impact on safety and optimal performance. By many accounts, the practice of medicine has lagged behind other fields in dealing with the complexities of human behavior in the workplace. ${ }^{7}$

Medicine should also take advantage of the integration of human factor and technical skill performance that is used by the AQP. Seldom does it seem that an optimal team atmosphere is established in the operating room. There are frequent problems with informing other team members of work overload or developing patient problems. Formal leadership is infrequently established for the procedure although, in discussions, assumptions were aired by both attending surgeons and anesthesiologists that they were de facto leaders. ${ }^{8}$ One result of the failure to establish leadership and to plan for alternative courses of action in the event of patient difficulties was frequent conflict. Anecdotally, most physicians and nurses can readily recount examples both of optimal teamwork and of equally impressive conflict from their own experiences. The advanced medical simulator in a high fidelity/realistic environment would be a wonderful tool for training the human factor team skills that are required by medical teams to perform at optimum levels of performance. This training would move beyond the anesthesiology resident to include nursing, surgical, and technical disciplines of study. This cross cultural training would bring together the individual technical skills of these groups to work together and build medical team concepts. For the anesthesiology resident, this simulation would provide the ability to induce and maintain a patient's anesthesia under different operating conditions-for example, interference from the surgeon or support staff during the intubation could be designed into the training. Having to intubate under unrealistic time pressure set by the surgeon to begin the procedure and how to manage this situation would be invaluable training. Cross cultural understanding of medical teams and how each group can be in a symbiotic relationship with each other to achieve a greater level of performance as a team would be an end level concept. For an effective integrated approach to simulator training there must be:

- specific training objectives established for the students using the simulation;

- training of the trainers to use the simulation and to present realistic scenarios to the students;

- specific criteria given to the evaluators to make their assessment of performance.

Medicine is at a point in its history where the use of advanced simulation is becoming a reality. With the cost and identification of medical errors increasing and advanced human simulation blossoming, simulation used in an integrated system design will provide a tremendous tool for training medical personnel. The study by Abrahamson et al ${ }^{1}$ was a beginning 35 years ago to provide an early but narrow assessment of the effectiveness of simulation. Although the 
conclusion was positive for the number of days in training and the proficiency achieved in a smaller number of trials, this is just the tip of the iceberg of how simulation can improve training.

Simulation is moving forward in medicine with advanced simulation technology being developed for ER, ICU, CCU, anesthesia, and many part task trainers for all disciplines of medicine. Simulation can fly in medicine if the medical disciplines will:

- Develop training that incorporates and uses simulation appropriate to the skills to be imparted: few training programs in today's world of medical training have assigned any knowledge or skill masteries to simulation.

- Create disciplined curricula which integrate technical and human factor performance based on risk and performance analysis: few programs in today's world of medicine assess the challenges in the operating environment of medicine to create effective training scenarios.

- Monitor program effectiveness and develop accountability for effective training: few programs in today's world of medicine assess training effectiveness and make dynamic changes as necessary to meet needs; remove the "we train this way because we always have" mindset.

- Measure and link performance improvement in medicine to the causal effects of training with simulation. Perform analysis and measure the ability of simulation to reduce medical error and the cost saving this provides. Training with simulation adds expense to a training program. Not only is the cost of the simulation an issue, but simulation requires a complete paradigm shift in the training program management. In today's world, medicine is taught as medicine is performed on the patient population, which is generating revenue. With simulation, both the house staff and residents must be pulled out of revenue patient care to work and study with simulation. This creates several challenges of staffing and time management which must be addressed if simulation is to be integrated into a medical training program. The complexity and expense of simulation has to be offset against the reduction of errors and associated cost.

It took aviation 35 years to develop and advance these training concepts. Medicine should capitalize on these years of experience to advance these concepts for the future use of effective simulation in medical training. It is time for medicine to take off, catch up, and move simulation forward into an integral systematic approach for training.

W R Hamman

United Airlines; rossha@aol.com

\section{REFERENCES}

1 Abrahamson S, Denson JS, Wolf RM. Effectiveness of a simulator in training anesthesiology residents. J Med Educ 1969;44:515-9.

2 NASA Dryden Flight Research Center. The role of aircraft simulation in improving flight safety through control training, NASA/TM-2002210731.Dryden Flight Research Center, Edwards, California, 2002.

3 Dillard AE. Validation of advanced flight simulators for human-factors operational evaluation and training programs, Foundations '02 V7V Workshop, John Hopkins University Applied Physics Laboratory, 2002.

4 Rolfe JM, Staples KJ, eds. 50 years of flight simulation. Conference Proceedings, April 1979.

5 Federal Aviation Authority (FAA). Line-oriented flight training programs, Advisory Circular 120-35, 1990.

6 Billings CE, Reynard WD. Human factors in aircraft accidents: results of a 7year study. Aviat Space Environ Med 1984;55:960-5.

7 Institute of Medicine. To err is human: building a safer health system. Washington, DC: National Academy Press, 2000.

8 Sexton JB, Thomas EJ, Helmreich RL. Error, stress, and teamwork in medicine and aviation: cross sectional surveys. BMJ 2000;320:745-9. 